\title{
Carnets
}

Revue électronique d'études françaises de l'APEF

Deuxième série - $16 \mid 2019$

Le Récit inachevé: études sur Mai 68

\section{Le néo-polar est-il un avatar littéraire de Mai 68 ?}

\section{Pierre-Michel Pranville}

\section{(2) OpenEdition}

\section{Journals}

\section{Édition électronique}

URL : http://journals.openedition.org/carnets/9769

DOI : 10.4000/carnets.9769

ISSN : 1646-7698

Éditeur

APEF

\section{Référence électronique}

Pierre-Michel Pranville, «Le néo-polar est-il un avatar littéraire de Mai 68 ? », Carnets [En ligne],

Deuxième série - 16 | 2019, mis en ligne le 31 mai 2019, consulté le 09 novembre 2019. URL : http:// journals.openedition.org/carnets/9769; DOI : 10.4000/carnets.9769

Ce document a été généré automatiquement le 9 novembre 2019.

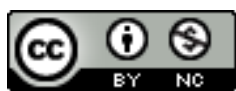

Carnets est mis à disposition selon les termes de la licence Creative Commons - Atribution - Pas d'utilisation commerciale 4.0 International. 


\title{
Le néo-polar est-il un avatar littéraire de Mai 68 ?
}

\author{
Pierre-Michel Pranville
}

1 Nous entendrons par avatar littéraire de Mai 68 une incarnation des idées de Mai 68 dans la littérature policière des années 70-80, incarnation qui donnerait à ce sous-genre une identité propre baptisée néo-polar par Jean-Patrick Manchette. La réponse est positive, mais c'est un oui nuancé, ou plutôt un « oui, mais pas seulement ». Oui, le néopolar va travailler des thèmes tels que la contestation de l'autorité, la remise en cause de la société de consommation, la revendication d'une liberté sexuelle et d'une contreculture. Le « oui mais pas seulement » doit être trouvé, au-delà des valeurs de Mai 68, dans le bilan que les auteurs du néo-polar tirent des événements auxquels ils ont, pour la plupart, participé. C'est ce que nous soulignerons après avoir étudié les quatre romans du corpus proposé. Nada de Jean-Patrick Manchette et Etat d'urgence de JeanFrançois Vilar, sont deux romans qui appartiennent à une veine néo-polar qui revisite l'histoire ; Suzanne et les ringards de Jean-Bernard Pouy et La Bête et la Belle de Thierry Jonquet explorent la société des oubliés, celle des saltimbanques et des cités HLM. Ces quatre romans représentent les deux grandes tendances que l'on peut identifier dans le néo-polar: la relecture de l'histoire également défendue par Didier Daeninkcx - nous avons à l'esprit Meurtre pour mémoire qui évoque les événements de Charonne, la répression d'une manifestation en 1961 où furent assassinés 400 algériens; l'autre tendance du néo-polar est, elle, résolument sociale, centrée sur les petits, les sansgrades et l'univers des cités ouvrières, ce qui est tout à fait nouveau en 1970.

Pour apprécier le caractère novateur de l'approche du néo-polar, il convient de faire un état des lieux de la littérature policière française des années 60 . Le roman policier dans sa composante Noir est une littérature de la crise, crise politique, économique, sociale et crise des valeurs. Il est issu du roman noir américain qui est né de la crise de 1929 et de la Prohibition, convoquant dans ses textes de nouvelles populations : les gangsters mais aussi les losers de la société. L'Etat et particulièrement la police ne garantissent plus la sécurité et la justice parce qu'ils sont corrompus. Manchette écrit :

Dans le roman criminel violent et réaliste à l'américaine. (Roman noir), l'ordre du

Droit n'est pas bon, il est en contradiction avec lui-même. Autrement dit le Mal 
domine historiquement. La domination du Mal est sociale et politique. Le pouvoir

social et politique est exercé par des salauds. (Manchette, $2003: 20$ ) institutionnel, sont désenchantés. C'est la Série Noire créée par Marcel Duhamel en 1945 qui domine le genre avec des traductions d'auteurs anglo-saxons et des romans noirs qui empruntent au milieu leurs gangsters et leur argot. Marcel Duhamel publiera Albert Simonin, Auguste Le Breton. En parallèle de la Série Noire, Boileau-Narcejac et Sébastien Japrisot s'orientent vers des romans privilégiant la psychologie et le rôle de la victime. Trois individualités se détachent du lot des publications policières : Georges Simenon et son commissaire Maigret, Frédéric Dard avec la série des San-Antonio, et Léo Malet qui créé le personnage de Nestor Burma. Enfin, surgit un auteur engagé qui détonne : Jean Amila que l'on peut considérer comme le précurseur du néo-polar par ses préoccupations sociales et son intérêt pour la relecture de l'histoire. Voilà où en est le roman noir en France à la veille de 1968.

«Avec le néo-polar, nous passons du roman voyou au roman engagé » (Schweighaeuser, 1997: 100). C'est la fin des gangsters romantiques et des commissaires infaillibles. Mais c'est aussi un coup porté aux privés torturés, derniers chantres de la Vertu, importés d'Amérique du nord. Patrick Manchette qui n'est pas seulement romancier, mais aussi chroniqueur et essayiste, a publié ses réflexions sur la littérature policière, et il explique ainsi son passage au néo-polar :

Comme j'étais totalement nourri de polars américains, pas du tout d'auteurs français, il me paraissait tout naturel, automatique, de suivre la voie des « réalistescritiques ». Le polar, pour moi, c'était, c'est toujours, le roman d'intervention sociale très violent. Je suis donc parti dans cette direction, où me poussait mon expérience de gauchiste (Manchette, $2003: 12$ ).

Ce roman d'intervention sociale va s'élever sur le fond contre les conformismes de la société et sur la forme contre les codes du roman policier français, codes venus d'Amérique. Sur le fond: moins de policiers, moins d'enquêtes, une dégradation des valeurs morales, des acteurs issus du peuple. Sur la forme: une écriture dépouillée, behavioriste, plus centrée sur les comportements que sur les états d'âme des personnages. Avec le néo-polar, la littérature policière va s'ancrer dans une vision sociale et politique très critique de la réalité française. Philippe Corcuff dans la revue Mouvements dit du roman noir en 2001 :

Le scepticisme à l'égard du monde et de ses conventions, comme des humains qui s'y " agitent ", l'ironie sombre à l'égard de soi (dans le cas du héros narrateur) et des autres, voire le cynisme et un relativisme immodéré (dans le «tout se vaut») sont souvent au rendez-vous. Une telle ambiance peut nourrir une radicalisation de la critique des institutions de nos sociétés (Corcuff, 2001 : 103)

Cette remarque s'applique également au néo-polar. Nous allons retrouver scepticisme, ironie, cynisme et relativisme dans chacun des romans de notre corpus. Le héros du néo-polar est un anti-héros, «il peut redresser quelques torts, mais il ne redressera pas le tort général de ce monde, et il le sait, d'où son amertume » précise Manchette.

\section{Qui est Jean-Patrick Manchette, l'auteur de Nada?}

7 Né en 1942 et décédé en 1995, il se définissait lui-même comme un ancien étudiant sans diplôme. Il écrit d'abord pour les autres, des scénarios; il traduit de l'anglais; il est 
critique pour Charlie Hebdo, et devient un théoricien du genre. En 1971, il publie Laissez bronzer les cadavres. Dans son dictionnaire, Claude Mesplède écrit :

Ce roman donne le ton de ce qu'on a appelé le néo-polar. Le monde exotique de Pigalle et de ses truands disparait au profit des classes moyennes, du retour à la nature et de la nostalgie de la révolution. Le roman noir devient social, critique de la vie quotidienne et résolument politique (Mesplède, 2003 : 289).

De ses dix romans, nous avons choisi Nada. Que raconte Nada? Un groupe constitué d'un prof de philo qui renonce à l'action armée pour des motifs doctrinaux, d'un anarchiste alcoolique, d'un garçon de café aigri, et de deux ex-activistes anarchistes projette d'enlever l'ambassadeur des Etats-Unis à Paris. Mais pourquoi faire ? Pour la publication d'un manifeste anti impérialiste ou pour la rançon? Contre toute attente, ces pieds-nickelés réussissent leur coup. Le commissaire Goémond qui les traque ne fera pas de prisonniers s'il les attrape. Seul Buenaventura, l'anarchiste catalan, échappera au siège de la police et rejoindra le philosophe pour le charger de dévoiler à la presse la brutalité de la police. Manchette veut signifier par là que les kidnappeurs sont finalement moins violents que la police, mais aussi que leur cause s'appuie sur un idéal peut-être, mais un idéal dont ils reconnaissent l'aspect dérisoire. Buenaventura s'enregistre pour la presse et avoue :

- J'ai fait une erreur, dit-il soudain. Le terrorisme gauchiste et le terrorisme étatique, quoique leurs mobiles soient incomparables, sont les deux mâchoires du... Il hésita.

- ... même piège à cons (Manchette, $1972: 164)$.

9 Le refus de l'autorité s'exprime dans l'enlèvement d'un ambassadeur américain, un topos appartenant à la geste révolutionnaire depuis celui de l'agent du FBI Dan Mitrione enlevé par les Tupamaros uruguayens en 1970, repris par Costa Gravas en 1972 dans Etat de siège, Yves Montand jouant le rôle de l'agent américain. Manchette dénonce la mâchoire étatique, manifeste dans la duplicité et le cynisme du Ministère de l'Intérieur qui conseille au policier de supprimer aussi l'ambassadeur, disparition que l'on mettra sur le compte du groupe Nada. Manchette déshabille l'Histoire et met quelques points sur les $\mathrm{i}$ en rappelant le climat de guerre des services entre les différentes polices et les organes de renseignement, en rappelant que certains d'entre eux étaient noyautés par l'extrême-droite représentée par un mystérieux Service d'Accentuation Civique, qui n'est autre que le SAC, Service d'Action Civique, réel celuici, une milice chargée des basses œuvres aux ordres de la droite: service d'ordre, pressions musclées, violences, voire assassinats. Reprenons la citation de Philippe Corcuff dans Mouvements :

Une telle ambiance peut nourrir une radicalisation de la critique des institutions de nos sociétés. Mais cette critique sociale semble hésiter entre la réouverture de l'espace des possibles face à la fermeture dominante du monde et à un fatalisme du « tout est gangrené » et du « à quoi bon » (Corcuff, 2003 : 103).

Dans le néo-polar, la réouverture vers des possibles est ténue. C'est plutôt no future. Dans Nada, sauf à un niveau individuel, dans la relation entre les personnages : Cash, la jeune passionaria du groupe, pratique une sexualité libérée dont bénéficiera Epaulard, le cinquantenaire, l'ex-activiste international ; et seule la confiance que confère l'amitié résiste face au rouleau compresseur de la raison d'Etat. Buenaventura n'a jamais douté de la loyauté de Treuffais, le prof de philo. Même s'il a renoncé à suivre le groupe dans l'action violente, il n'a pas parlé, il n'a pas trahi, le catalan en est persuadé. Au niveau 
collectif, seule la presse ressort indemne de la lessiveuse Manchette. Elle est le dernier recours qui garantit l'éclatement de la vérité.

\section{Etat d'urgence de Jean-François Vilar}

11 Né en 1947 et mort en novembre 2014 à Paris, Jean-François Vilar a écrit sept romans et une douzaine de nouvelles. Comme Manchette, ce n'est pas un auteur prolifique. Vilar a créé un personnage récurrent, Victor Blainville, photographe dans un grand quotidien, un poste d'observation privilégié de ses semblables; c'est un soixante-huitard comme son créateur. Dans Etat d'urgence, Blainville, curieusement, n'a qu'un petit rôle. Il délègue ses fonctions de fouineur à Adrian Leck, cinéaste inégal qui projette un film retraçant l'itinéraire d'un repenti des Brigades rouges, Arno, qui l'accompagne en Italie sous une nouvelle identité. Délaissant Paris, Vilar a choisi Venise, et pas n'importe quand, pendant le Carnaval, comme métaphore d'un monde fou. La fête masque et dévalorise les enjeux d'une nouvelle confrontation entre la police et les terroristes qui menacent de faire sauter le Grand Canal. Pourquoi Leck fouine-t-il ? Il cherche à s'approprier les lieux, les témoins, les raisons qui ont entrainé Arno dans la lutte armée. Il va être servi. Sa rencontre, un flirt, avec la juge Vanese, une promesse de sexe libre, qui va tourner court: Vanese se fait descendre dans ses bras. Leck récupère des dossiers de la juge qui vont lui ouvrir les yeux sur le passé d'Arno, sur son retour aux affaires aux côtés du commissaire Gozzi, sur les liens de son producteur vénitien avec la mafia, sur les manipulations de la police et surtout sur les intérêts communs que partagent la mafia et les Brigades rouges. Leck lit le dossier d'Arno :

J'ai réfléchi longuement et sereinement à mon parcours au sein de l'organisation clandestine. (...) Malgré son intensité, le débat qui y a eu lieu fut tel que nous avons perdu de vue ce pourquoi l'organisation était née. L'enthousiasme qui m’avait porté à combattre pour la classe ouvrière m'avait fait perdre de vue la classe ouvrière elle-même (...) Je n'arrivai plus à voir que notre lutte, malgré notre bonne foi, se transformait en une guerre de bandes, une guerre entre nous, entre les BR et l'Etat (Vilar, 1996 : 35).

Le groupe Nada de Manchette a également ressenti cette rupture avec ceux pour lesquels il est censé lutter au moment de la condamnation de l'enlèvement de l'ambassadeur par les partis représentant la classe ouvrière, et il ne l'a pas comprise. Dans Nada comme dans Etat d'urgence, le débat d'idées, le combat politique a laissé la place à une traque, à une guerre sans fin dont la seule motivation est sa pérennité. Adrian Leck séjourne chez son producteur dans un somptueux palais qui abrite un microcosme culturel isolé de la réalité. Vilar ne se prive pas de critiquer le monde artistique, ses mœurs dépravés, ses liens avec d'anciens nazis et avec la mafia qui finance les activités culturelles, dont son film. Mais Adrian Leck, dont le passé de militant n'est pas si éloigné que cela, aime se frotter au terrain, ce qui lui vaudra d'être kidnappé par les Brigades rouges et de passer de l'autre côté de la caméra dans une séquence d'autocritique non dénuée d'une sincère auto-dérision. L'auto-dérision est aussi un des marqueurs des protagonistes du néo-polar.

13 L'issue du roman est plus noire que celle de Nada. La fin, un peu rocambolesque où des brigadistes veulent faire sauter un tanker face à la place Saint Marc, est un véritable bain de sang. Leck survit mais il a perdu son inspirateur Arno, et son film. Le commissaire Gozzi survit, mais ce n'est pas grâce à lui que le drame a été évité mais grâce à la mafia. La guerre va donc se poursuivre, avec d'autres combattants, sur 
d'autres fronts. Jean-François Vilar a revisité l'histoire des Brigades rouges avec une précision chirurgicale, un réalisme qui ne peut être que le fruit d'une documentation poussée. Son diagnostic sur le terrorisme est le même que celui de Manchette : un vaste gâchis dont il tire un profond désenchantement.

\section{Suzanne et les ringards, le deuxième roman de Jean- Bernard Pouy}

14 Ce roman appartient à la seconde grande tendance du néo-polar, celle qui parle des marginaux, des populations des cités. Etudiant en histoire de l'art, professeur de dessin, journaliste puis romancier, Claude Mesplède dit de Pouy qu'il est un auteur inclassable. Qui s'impose des contraintes formelles comme par exemple le personnage d'une princesse dans Suzanne et les ringards, ou comme des jeux avec l'alphabet, ou des citations inventées. Qui joue avec la langue en usant des néologismes et des calembours. «Cet aspect ludique imprègne son œuvre même lorsque le ton se veut plus grave» précise Mesplède (Mesplède, 2003: 563). Contrairement aux deux auteurs précédents Pouy a publié plus de 50 romans et autant de nouvelles ou de contributions à des ouvrages collectifs, dont Mes soixante huitres.

15 Qui est la Suzanne de Suzanne et les ringards? C'est une petite groupie d'un ensemble de rock qui parvient à passer la soirée avec ses musiciens préférés, les ringards du titre. Voici son portrait :

La chair à star... Chair pauvre.

- Ecoutez-moi : je fais mes trente-neuf heures dans un entrepôt de cartonnage. Debout. Je ne vois rien d'autre. Après... Chez moi. J'ai dix-huit ans. Mes copains ne sont pas plus intéressants que moi... Mes parents sont gentils, c'est tout. Je ne me sens pas comme eux. La vie a déjà un sale goût... Laissez-moi cette soirée. Peut-être y'en aura d'autres, peut-être pas. Laissez-moi cette soirée (Pouy, 2005 : 26).

Gaddes, l'homme à tout faire, se laisse attendrir et la présente au groupe avant de quitter l'hôtel. Elle sera retrouvée morte au matin dans la chambre de Gaddes, justement, le logisticien et gros bras de l'équipe, un colosse et ex militant gauchiste.

La fiche des RG, membre actif d'un obscur groupuscule qui a eu sa part dans les désordres, et puis la dérive, les grèves, le refus du travail, de l'armée, des impôts. Trois ans de taule pour un cassage de gueule syndicale fasciste. Et puis plus rien sur la fiche : ne pouvait y figurer que l'immense dégoût de voir mes amis s'effilocher, changer et m'exclure, la surprise de constater qu'en trois ans, les têtes tournent, changent et défendent petit à petit tout ce qu'elles ont abhorré (Pouy, 1985 : 40).

Après le portrait de Suzanne, gentille ouvrière qui veut rêver un peu, voici le parcours type d'un ouvrier, ex- activiste gauchiste, déçu, amer et résigné. Gaddes est surnommé Dumbo parce qu'il a une énorme tache lie-de-vin sur la partie droite du visage. La tournée se poursuit mais Dumbo n'aura de cesse de découvrir qui a tué Suzanne. « T'es un zorro de merde » lui lancera un des musiciens, agacé par ses questions. Gaddes les déteste, sauf Lucie, l'ingénieure du son. Au même moment, à l'autre bout du pays, une très jeune femme, Valérie $\neg$ la fameuse contrainte de la princesse $\neg$ pleure son amant et metteur en scène qui vient de se faire tuer dans ses bras. Il reste au lecteur à attendre que les deux histoires se rejoignent. Gaddes abandonne le groupe, dégoûté par leur cynisme et leur égoïsme pour atterrir dans un petit village de l'Ardèche où a échoué Valérie, l'actrice en rupture de ban. Une rencontre fusionnelle, sexuelle, au bord de la rivière, quand deux tueurs veulent s'en prendre à la star. Gaddes va la sauver en les 
éliminant. Reconnue, Valérie est happée par les journalistes et retourne à son milieu. Et Gaddes, à sa quête : qui a tué Suzanne?

Mais j'en avais plus rien à tordre. Tout ça, c'était de la vie, du bonheur, du rassurant. Moi, je roulais, je m'enfonçais pertinemment dans du malheur, dans la mort, dans l'absurde vengeance, vers cinq jeunes rockers, vers un manager, vers un producteur, peut-être plus haut, vers une société, un groupe, une multinationale. Ça aurait pu être un Etat, je m'en foutais, je fonçais, je m'enfonçais, je profitais de cette énergie noire qui me balayait le corps, en haut en bas, de droite à gauche, de devant en arrière.

Folie. Rage. Folirage (Pouy, 1985 : 162).

Il découvrira, ultime trahison, que c'est Lucie, sa seule amie, qui a causé la mort de la petite groupie lors d'une séquence drague trop appuyée. Dans cette road story qui donne la part belle à l'univers de la route la nuit, Pouy peint au vitriol le milieu du rock et aussi celui du cinéma. La solitude, les trahisons, le sexe hétéro et homosexuel, l'individualisme, la dénonciation d'un monde artistique où les enjeux commerciaux l'emportent. Tout cet ensemble compose un polar social, critique, engagé. Et désenchanté.

\section{La Bête et la Belle de Thierry Jonquet}

Thierry Jonquet est né à Paris en 1954 et mort en 2009. Il devient ergothérapeute après des études de philosophie, puis enseignant en milieu carcéral. Ses premiers romans et plusieurs autres sont tirés de son expérience hospitalière. La vengeance est un thème récurrent chez lui, dans tous ses textes. Il est l'auteur d'une quinzaine de romans et autant de polars pour la jeunesse, d'une cinquantaine de nouvelles et autres contributions collectives. Il s'inspire souvent de faits divers comme c'est le cas dans $L a$ Bête et la Belle. Mais qui est donc la Bête ? C'est Le Coupable, un professeur de collège qui assassine à tour de bras ceux dont il pense qu'ils ont découvert une partie de son secret. Et ce sont des crimes presque parfaits. Et qui est la Belle? La première victime du roman, fictive, celle-ci. C'est Irène, la documentaliste du collège, qui a partagé la vie de la Bête, peu de temps, avant de fuir. Il l'a tuée, dit-il, et pliée dans son congélateur, mais c'est faux. Pour masquer le congélateur, il va l'entourer de sacs poubelles qui vont peu à peu envahir tout l'appartement, voilà le secret. Le commissaire Gabelou dispose des aveux du Coupable, enregistrés sur un magnétophone, mais c'est insuffisant. Il veut obtenir le témoignage de Léon, un vieil SDF qui a cohabité avec le professeur et dont il est le seul ami. Mais Léon est loyal et ne dira rien.

Jonquet introduit dans le néo-polar des constructions narratives sophistiquées: Le commissaire à la $3^{\text {ème }}$ personne et Léon à la $1^{\text {ère }}$ personne se partagent les perspectives ; la gestion d'un suspens par l'annonce de l'entrée en scène des protagonistes tous nommés par leur rôle dans le roman: Le Coupable, le Gamin, la Vieille, le Commis, le Visiteur, en fait, l'assassin et ses quatre victimes. Et l'Emmerdeur, l'agent de la compagnie d'assurance. Échappent à cet étiquetage : Irène, la sulfureuse documentaliste, Léon, le SDF, et Gabelou le commissaire. L'intrigue se déroule pratiquement en huis clos dans l'appartement du professeur devenu Diogène. Un appartement situé dans le HLM d'une cité nouvelle au nom imaginaire, Altay 2, un habitat populaire où tous travaillent à l'usine Citroën en grève et où les gamins vont au CES, le Collège d'enseignement général : 
Ce n'était que saloperies de tubulures métalliques hachurant l'alignement de vitres sales : une grille faisait le tour de la cour, et, près de la loge du concierge, une sculpture anémique de béton armé, représentant un gros poisson, n'en finissait plus d'agoniser en silence, la bouche grande ouverte...

Gabelou avait secoué la tête, en songeant que ce n'était pas avec une horreur pareille que les gosses une fois adultes, pourraient sombrer dans des nostalgies d'enfance.

Les bâtiments de la cité faisaient face au collège. Couverts d'affiches syndicales qui protestaient contre les licenciements de l'usine Citroën, ils étaient tous semblables, attentivement crépis d'ocre ou de bleu (Jonquet, 1985 : 54).

21 Nous sommes dans la vraie vie. Jonquet décrit les HLM de la première vague, 1960-1970, construit à la hâte contre les villages entourant Paris, habités par les ouvriers et les employés qui achètent tout au Carrefour du coin, les professeurs qui achètent tout à la Camif (la coopérative d'achat des enseignants), bref une toute petite bourgeoisie qui accède à la consommation. La satire sociale est féroce.

Cette société d'une extrême banalité que décrit Jonquet est donc capable des crimes les plus gratuits, les plus violents. C'est ce qui fait la noirceur de ce roman: la transgression des valeurs les plus élémentaires par un représentant de ceux qui constituent le socle de la République, un professeur de collège. Et, collectivement, il n'y a point de salut. La seule ouverture vers des possibles plus attrayants est une nouvelle fois individuelle : c'est l'amitié, ici des amitiés inattendues entre un professeur coincé et un SDF, entre le vieux flic bourru et ce SDF. Encore faut-il accepter les mains qui se tendent. Léon, le SDF préférera le suicide.

Pouvons-nous conclure que le néo-polar a trouvé sa source dans les valeurs de Mai 68 ? Oui, la contestation de l'autorité, la remise en cause de la société de consommation, la revendication d'une liberté sexuelle et d'une contre-culture sont présents chez les auteurs étudiés et constituent par leur récurrence une identité littéraire que Manchette a nommé néo-polar. Ernest Mandel, lui, parle carrément de « polar révolutionnaire » ou de « polar prolétarien » dans le sens où :

La nature du genre, celle d'une remise en question radicale de la société dans son ensemble, de l'Etat et de ses appareils, y compris la police, y compris les détectives privés (...) C'est la violence institutionnelle quotidienne - ou, si l'on veut, le terrorisme d'Etat - qui est catégoriquement dénoncée, à laquelle s'oppose l'insignifiante mini-violence des laissés-pour-compte (Mandel, 1986 : 167).

Le contrat est rempli, mais il y a plus. Le désenchantement que nous avons observé et qui sourd de tous ces textes noirs, pessimistes. Il rejoint « le fatalisme du «tout est gangrené », du «tout se vaut » selon l'expression de Philippe Corcuff. Ernest Mandel, faisant allusion au roman Nada y voit une contradiction entre l'intention et le texte, et il est sévère :

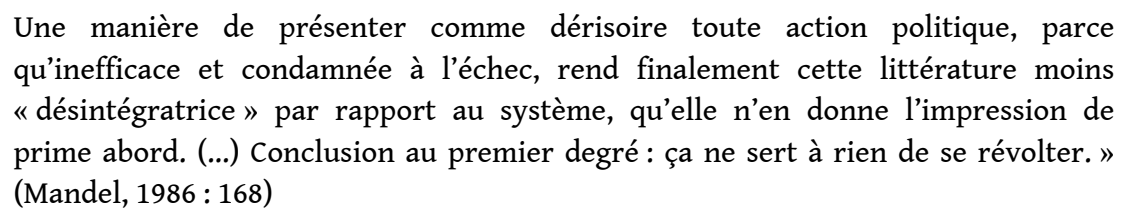
qu'inefficace et condamnée à l'échec, rend finalement cette littérature moins «désintégratrice» par rapport au système, qu'elle n'en donne l'impression de prime abord. (...) Conclusion au premier degré : ça ne sert à rien de se révolter. » (Mandel, $1986: 168$ )

Il est dans son rôle de théoricien marxiste. Voyons si ce désenchantement est représentatif de l'état d'esprit des auteurs du néo-polar, s'il est un autre marqueur de ce sous-genre littéraire et pourquoi. Dans un article intitulé «Le néo-polar, du gauchisme politique au gauchisme littéraire », Annie Collovald et Éric Neveu lient les 
itinéraires personnels des auteurs et leurs prises de position littéraires. Parlant de Daeninckx, Fajardie, Jonquet, Pouy et Vilar :

Tous les cinq sont venus du gauchisme de l'après 68 , et, depuis leur entrée en littérature noire dans les années 1979-1984, tous ont conservé un regard attentif aux plus démunis et rebelle face à l'ordre social et politique établi. Leurs transformations en écrivains professionnels ne semble pas les avoir fait renoncer à leurs idéaux anciens (Collovald-Neveu, $2001: 78$ ).

Ces deux chroniqueurs parlent d'une offre de continuation de l'esprit gauchiste en littérature. Chronologiquement, elle tombe bien, à un moment où le public se lasse des polars classiques, où ils sont à la recherche d'une démarche "plus intellectuelle (humour, complexité des intrigues, auto-références...) et plus orientée vers une réflexion sur le monde social contemporain ". C'est un monde qu'ils connaissent bien de par leur passé professionnel souvent hétéroclite et au contact des populations les plus défavorisées. Il y a donc dans leur volonté d'écrire celle de rester fidèle à leurs engagements et de continuer leur combat.

Mais et le désenchantement? Dans plusieurs entretiens, Dominique Manotti, romancière, auteure de nombreux polars sociétaux ou géopolitiques, rappelle que Mai 68 qui a été une réussite dans l'évolution des mœurs est un échec politique. C'est cet échec politique qui a fonctionné comme un aiguillon pour le néo-polar. Elle dit: «j'écris parce que je ne crois plus à la révolution. Je n'ai pas pu la réaliser : je l'écris ». Cette prise de position nette a été amplifiée par la déception de l'après-Mitterrand. Mitterrand arrive au pouvoir en 1981, les espoirs de la gauche de la gauche se sont envolés dès 1982, avec le retour à une réalpolitique. C'est cet échec du mouvement de 68 plus que les valeurs qu'il porte et qu'ils ont largement contribué à faire connaitre qui taraude les auteurs du néo-polar, la conscience d'être passé très près d'un possible révolutionnaire maintenant inaccessible. Et c'est ce sentiment d'échec et de culpabilité, ce désenchantement qui est partagé dans ce sous-genre de la littérature policière.

Le néo-polar a-t-il survécu aux années 1980-90 ? La réponse est oui. Un des outils de la pérennité de « l'esprit néo-polar » est la collection Le Poulpe, fondée par Jean-Bernard Pouy, Patrick Raynal et Serge Quadruppani en 1995. L'idée ? Un jeu littéraire collectif très important, cet approche collective, issue des valeurs de 68 - avec une «bible ", un cahier des charges: un auteur différent pour chaque volume; un titre qui soit un calembour - le premier titre est de Pouy : La petite écuyère a cafté, pour la petite cuillère à café ; des personnages imposés avec un héros récurrent, le détective libertaire Gabriel Lecouvreur, dit Le Poulpe, avec Vergeat un clodo omnipotent, avec la fiancée du Poulpe, la coiffeuse Cheryl, et avec Pedro, l'ex-activiste anarchiste. C'est un succès. La collection qui a fêté ses 20 ans en 2015 a compté 178 titres à cette date. Tous les auteurs de noir qui comptent aujourd'hui sont passé par Le Poulpe et sa "bible", et par son esprit soixante-huitard: Lecouvreur est un zorro moderne, un redresseur de tort qui dénonce les injustices et défend les laissés-pour-compte. Et le seul survivant de nos quatre romanciers, Jean-Bernard Pouy poursuit son travail d'écriture avec acharnement, un acharnement orienté contre l'ordre établi, comme dans Ma ZAD, son dernier roman paru en 2018, toujours à la Série Noire. ZAD comme zone à défendre, comme $\neg$ quelle coïncidence $\neg$ la ZAD de Notre Dame des Landes défendue par de nombreux militants qui a permis d'éviter la construction d'un aéroport dans le bocage nantais. Il est vrai que 50 ans après 1968 , les sources d'inspiration chères au néo-polar ne manquent pas. 
-Bernard Pouy avait publié à l'occasion des 40 ans de Mai 68 un billet d'humeur intitulé Mes soixante huîtres. Il y décrit un déjeuner dominical, de ces repas de famille honnis quand on est jeune : un couple, la soixantaine, et ses deux enfants, la trentaine, accompagnés de leurs conjoints. Le repas tourne au débat entre le père ex-soixantehuitard et ses enfants qui sont devenus des cadres créatifs épanouis, un peu écolos, un peu de gauche, bref, bobos. Le débat tourne au vinaigre comme tous les dimanches, mais là, le père s'emporte, va chercher son vieux sac à dos et, avant de prendre la fuite, délivre aux siens une tirade mémorable qui est un condensé de tous les espoirs et toutes les désillusions engendrées par ce printemps de mai. En voici un extrait :

Vive la société ludique! Vive les mômes et les voyous! Abolition de la société de classes! Autogestion de la vie quotidienne! Ici, bientôt de charmantes ruines. Merde au bonheur! Vivez! N'admettez plus d'être immatriculés, fichés, opprimés, réquisitionnés, prêchés, recensés, traqués! Chassez le flic de votre tête! Mangez vos professeurs! N'obéissez jamais plus! Ne travaillez jamais! Ne faites jamais confiance à quelqu'un de plus de trente ans! Ne prenez plus l'ascenseur, prenez le pouvoir! Que c'est triste d'aimer le fric. Brûlez les banques! Vous êtes creux. Vous finirez tous par crever du confort. La vie est ailleurs. Soyez contre tout. Surtout contre vous-même. Oubliez tout ce que vous avez appris. Commencez par rêver. Prenez vos désirs pour des réalités. Soyez réalistes, demandez l'impossible. Ouvrez les fenêtres de votre cœur. Faites la somme de vos rancœurs et ayez honte. $\mathrm{Ne}$ gardez pas votre sang-froid. Jouissez sans entraves! Déboutonnez votre cerveau aussi souvent que votre braguette ! En tout cas, pas de remords! (Pouy, $2008: 27$ )

Le néo-polar n'est pas un manifeste tardif des idéaux de 68. Il exprime le désespoir de ceux qui y ont crus en inscrivant leur lutte dans la littérature noire.

\section{BIBLIOGRAPHIE}

COLLOVALD, Annie, NEVEU, Éric (2001). « Le Néo-polar, du gauchisme politique au gauchisme littéraire », in Sociétés et représentations. 2001/1 ( $\left.n^{\circ} 11\right)$ DOI 10.3917/sr. 011.0077. 27)

CORCUFF, Philippe (2001) « Désenchantement et éthique » in Mouvements, Le polar entre critique sociale et désenchantement, $\mathrm{n}^{\circ}$ 15/16, juillet-aout 2001.

JONQUET, Thierry (1985). La Bête et la Belle. Paris : Gallimard, « Folio Policier ».

MANCHETTE, Jean-Patrick (1972). Nada. Paris : Gallimard, « Carré Noir ».

MANCHETTE, Jean Patrick (2003). Chroniques. Paris : Rivages « Noir».

MANDEL, Ernest (1986), Meurtres exquis. Histoire sociale du roman policier, Paris : Editions La Brèche.

MESPLEDE, Claude (2003). Dictionnaire des littératures policières. Nantes : Joseph K.

POUY, Jean-Bernard (1985). Suzanne et les ringards, Paris : Gallimard, « Folio Policier ».

POUY, Jean-Bernard (2008). Mes soixante huîtres, Montreuil : Folies d'encre.

SCHWEIGHAEUSER, Jean-Pierre (1997). « Le roman noir » in Les Temps modernes. Paris, nº 595.

VILAR, Jean-François (1996). Etat d'urgence, Paris : Editions J'ai lu.

Carnets, Deuxième série - 16 | 2019 


\section{RÉSUMÉS}

Parmi les nombreux sous-genres du roman policier français contemporain, le néo-polar est une perle rare parce qu'il n'est pas courant qu'un mouvement social donne naissance à un avatar littéraire. Avatar, il l'est pour deux raisons. D'abord, il adhère aux valeurs de Mai 68: la contestation de l'autorité, la remise en cause de la société de consommation, la revendication d'une liberté sexuelle et d'une contre-culture. Nada de Jean-Patrick Manchette et Etat d'urgence de Jean-François Vilar, sont deux romans qui appartiennent à une veine néo-polar qui revisite l'histoire. Ensuite, il y a le temps du constat, et les romanciers qui ont pour la plupart participé aux événements sont sévères et l'expriment dans Suzanne et les ringards de Jean-Bernard Pouy et La Bête et la Belle de Thierry Jonquet: que reste-t-il de Mai 68 ? Cette seconde veine est sociale, amère. Les écrivains-militants, déçus, continuent un combat inachevé en décrivant les banlieues, les usines et les vies que l'on y mène.

Among the many secondary genres of the contemporary French detective story, the Neo polar is a real gem because it is not common when a social movement gives birth to a literary avatar. It is an avatar for two reasons. First it adheres to the values of May 68: challenging authority, questioning the consumer society, claiming sexual freedom and counter culture. Nada by J.P Manchette and État d'urgence by J.F Vilar are two novels which belong to a Neo whodunit inspiration which reinterprets history. Then comes the time of acknowledgment and the novelists who, for most of them, took part in the events are harsh critics and express it in Suzanne et les ringards by J.B Pouy and la bête et la belle by T. Jonquet: what is left of May 68.? This second inspiration is social and bitter. The disappointed activist writers keep fighting by describing the suburbs, the factories and the lives that are led there.

\section{INDEX}

Mots-clés : littérature, Mai 68, Néo-polar, espoir, désillusion

Keywords : literature, May 68, Neo polar, hope, disillusion

\section{AUTEUR}

PIERRE-MICHEL PRANVILLE

CREPAL Sorbonne Nouvelle Paris 3

piermichelpranville[at]free.fr 